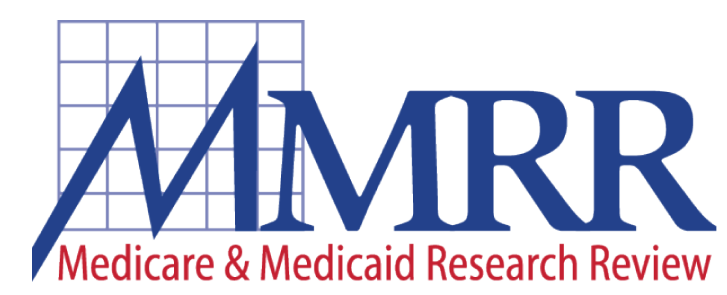

2013: Volume 3, Number 4

A publication of the Centers for Medicare \& Medicaid Services, Office of Information Products \& Data Analytics

\title{
Telehealth and Medicare: Payment Policy, Current Use, and Prospects for Growth
}

\author{
Matlin Gilman and Jeff Stensland \\ Medicare Payment Advisory Commission
}

Objective: Evaluate the growth in various types of Medicare-paid telehealth services.

Background: There has been a long-standing hope that telehealth could be used to reduce rural patients' travel times to specialty physicians. Medicare covers telehealth services provided through live, interactive videoconferencing between a beneficiary located at a certified rural site and a distant practitioner.

Methods: We analyzed 100\% of telehealth Medicare claims for 2009 matched to individual patient ZIP codes and individual provider characteristics.

Results: Despite increases in Medicare payment rates for telehealth services, expansions of covered services, reductions in provider requirements, and provisions of federal grants to encourage telehealth, growth in adoption of telehealth among providers has been modest. Medicare claims indicate that only 369 providers had 10 or more Medicare telehealth consultations in 2009. Roughly half of the 369 were mental health professionals, and about one-in-five of the 369 were non-physician professionals (e.g., physician assistants and nurse practitioners). On balance, the strong areas of telehealth are mental health and, surprisingly, nonphysician professionals. The comparative advantage of mental health could be the verbal (rather than physical contact) nature of mental health care, and the comparative advantage of non-physician professionals could be their lower labor costs.

Keywords: Telehealth, telepharmacy, tele-emergency care, Medicare payment policy

ISSN: 2159-0354

doi: http://dx.doi.org/10.5600/mmrr.003.04.a04 


\section{Medicare \& Medicaid Research Review} 2013: Volume 3, Number 4

\section{Mission Statement}

Medicare \& Medicaid Research Review is a peerreviewed, online journal reporting data and research that informs current and future directions of the Medicare, Medicaid, and Children's Health Insurance programs. The journal seeks to examine and evaluate health care coverage, quality and access to care for beneficiaries, and payment for health services.

\section{http://www.cms.gov/MMRR/}

\section{U.S. Department of Health \& Human Services Kathleen Sebelius Secretary}

\section{Centers for Medicare \& Medicaid Services Marilyn Tavenner Administrator}

Editor-in-Chief

David M. Bott, Ph.D.

The complete list of Editorial Staff and Editorial Board members may be found on the MMRR Web site (click link): $\underline{\text { MMRR Editorial Staff Page }}$

Contact: $\underline{\text { mmrr-editors@cms.hhs.gov }}$

Published by the Centers for Medicare \& Medicaid Services.

All material in the Medicare \& Medicaid Research Review is in the public domain and may be duplicated without permission. Citation to source is requested.

\section{Introduction}

There has been a long-standing hope that telehealth could be used to overcome a lack of local medical specialists in rural areas. To support rural access to care, Medicare covers telehealth services provided through live, interactive videoconferencing between a beneficiary located at a certified rural site and a distant practitioner. These services include office psychiatry visits, other office visits, and additional physician visits (e.g., consultations). Since 1999, Medicare payment rates for telehealth services have increased, the number of telehealth services that Medicare covers has increased, provider requirements have been reduced, and federal grants supporting telehealth adoption have been provided. The objective of this study is to evaluate growth in various types of Medicare-paid telehealth services. We find that despite higher payments and lower regulatory burdens, growth in adoption of telehealth among providers has been modest.

\section{Background}

\section{Evidence of Impact of Telehealth on Health Outcomes}

The Agency for Healthcare Research and Quality assessed the body of literature on the efficacy of telehealth for the Medicare population, and found that telehealth was most effective for specialties that rely on verbal interactions and not necessarily physical contact, including mental health and neurology (Hersh et al., 2006). For such specialties, care provided through telehealth can probably achieve results comparable to care provided in person. There was mixed or limited evidence on the efficacy of telehealth in other specialties, including dermatology, ophthalmology, and wound care. 
More recently, Richardson and colleagues examined the body of literature on telemental health and also concluded that the treatment of many mental health conditions through telehealth can achieve clinical outcomes comparable to those achieved when the same treatments are provided face-to-face (Richardson, Frueh, Grubaugh, Egede, \& Elhai, 2009). Their conclusion was drawn in part from the largest randomized and controlled telemental health trial (O'Reilly et al., 2007). The trial randomly assigned 495 patients (ages 18 to 65) to receive up to four months of psychiatric consultation through telehealth or face-to-face care. The most prevalent condition was depression. On all outcome measures (functioning, hospital admissions, and mental health severity scores), telehealth achieved clinical outcomes equivalent to those achieved through face-to-face care. In addition, patient satisfaction was similar between the groups.

Despite literature showing that telehealth can achieve equivalent outcomes for many types of services, there were only 26,000 telehealth visits in 2006, which is less than one visit for every 300 rural Medicare beneficiaries (Hartstein, Warren, \& Howe, 2011). The literature cites several reasons for the limited use of telehealth. Common explanations include the lack of universal private pay coverage, thereby discouraging capital investment in telehealth; interstate licensure issues; nonuniform engineering standards; confidentiality and liability concerns; and, in some cases, a perceived lack of need for telehealth services (Abel et al., 2005; Institute of Medicine, 2004; Johnston, Weeler, Deuser, \& Sousa, 2000; Luo, 2008; Whitten \&Buis, 2006). In addition, would-be distant practitioners may consider providing telehealth services to be a poor investment of their time (Grigsby et al., 2007). Practitioners with a full workload may decide that telehealth requires more time and effort than they are willing to commit. In addition, telehealth introduces a disruption to usual practice patterns, and practitioners may not be inclined to adjust their routine to accommodate telehealth. The cost of managing the daily operation of video networks; the cost of peripheral devices, such as dermatology cameras and digital stethoscopes, and prior adverse experiences in telehealth, such as scheduling issues, cancellations, and technical difficulties with videoconferencing, also may discourage the adoption of telehealth (Luo, 2008). Providers may not want to deal with these administrative difficulties if they already have a sufficient population of local patients.

The discussion in the literature often focuses on the lack of specialty care in rural areas. The general assumption is that telehealth will be used to bring specialists (e.g., dermatologists, nephrologists, psychiatrists) with a higher level of training to rural areas via teleconferencing. A clearly missing part of the literature is a systematic analysis of the degree to which nurse practitioners and physician assistants are delivering care via telehealth.

\section{Literature on Two Emerging Uses: Telepharmacy and Tele-emergency Care}

The literature suggests promise for the use of telepharmacy and tele-emergency care, where consulting pharmacists supervise remote pharmacy technicians in rural pharmacies and hospitals, and where central emergency room physicians consult with remote primary care providers treating patients in emergency rooms. The Commonwealth Fund conducted a report on North Dakota's experience with telepharmacy and found that telepharmacy extended access to patients in their rural communities and was economically sustainable (McCarthy, Nuzum, Mika, Wrenn, \& Wakefield, 2008). Initiated in 2002 and still in operation today, the North Dakota 
Telepharmacy Project features live, interactive videoconferencing that enables pharmacists at central sites to supervise pharmacy technicians at remote sites, provide patient counseling, and order, verify, and approve prescriptions. One pharmacist in a central location can supervise several retail telepharmacy sites and hospital-based pharmacies. At the time when the study was conducted, over 50 retail telepharmacy sites and 25 critical access hospital sites in North Dakota were receiving pharmacist support via teleconferencing. These telepharmacies were formed with much lower fixed costs and were operating in rural communities where populations were too small to support a traditional pharmacy. While there is no direct payment from Medicare for telepharmacy, remote retail sites generated enough additional revenue through pharmaceutical sales to fund their costs and the time of the supervising pharmacist. All remote pharmacy sites became self-sustaining after their first year of operation, and over the course of the project, none of the remote sites closed (C. D. Peterson, personal communication, August 29, 2011). The net result is that patients in small towns benefitted from pharmacist staff without having to have the patient volume in a single site to support a pharmacist. Telepharmacy could represent a promising way to give residents of small rural communities not only access to pharmaceuticals, but also access to pharmacist expertise.

The literature also suggests some promise for tele-emergency care, which makes emergency medicine expertise and support available to patients and practitioners at small rural hospitals. One potential reason for expansion of the teleemergency care in small rural hospitals is the rapid conversion of small rural hospitals to critical access hospital status from 1999 to 2006. This could have led to increased use of Telehealth by 2009 , because critical access hospitals are reimbursed their allowable costs of care, including the stand-by cost of tele-emergency equipment in their emergency rooms.

Some rural emergency departments are using telehealth for rapid consultation with emergency care specialists at distant sites. While studies on telehealth in emergency departments tend to be conducted by researchers associated with teleemergency care programs, the results from these studies are generally positive (Blanchet, 2008; Doheny-Farina et al., 2003; Duchesne et al., 2008; Latifi et al., 2007; Ricci et al., 2003; Rogers et al., 2001; Sorondo, Holmberg, \& Bjorn, 2011).

Duchesne and colleagues examined the impact of telehealth on trauma care provided at seven rural hospital emergency departments in Mississippi (Duchesne et al., 2008). The study sample included over 800 traumatically-injured patients presented to the participating hospital emergency departments between January 2000 and January 2005. These patients were grouped according to whether they were presented before or after the initiation of the telehealth program in mid-2002. In the period before the telehealth program, the hospitals provided initial patient evaluations without assistance from the trauma center and then transferred the patients to the trauma center for definitive management. In the period during the telehealth program, initial evaluations were provided with assistance from physicians at the trauma center via telehealth. Telehealth enabled audio-visual, real-time communication between the nurse practitioners and patients at the hospitals and the physicians at the trauma center. The researchers found that, on average, patients who received telehealth-assisted care had significantly shorter lengths of stay at the hospitals and were less likely to be transferred to the trauma center than patients who did not. Because most of the patients who received telehealth-assisted care 
were able to avoid being transferred to the trauma center, they may have incurred lower costs than patients who did not. These findings suggest that tele-emergency care may improve appropriateness of care through improving access to specialists at trauma centers and may also save money through avoiding expensive transports.

\section{Past Medicare Policy Changes: Payments Are Up, Regulatory Burdens Are Down}

In January 1999, legislation allowed for Medicare to begin paying for telehealth with a single payment set to the physician fee schedule rate and split between the distant practitioner, who would receive 75 percent of the fee, and the practitioner at the originating site (that is, the site where the patient is located) who would receive the remaining 25 percent. At that time, Medicare payment policy surrounding telehealth was considered limiting in several regards. First, originating sites were required to have a practitioner (e.g., physician, nurse practitioner) present with the beneficiary during telehealth visits. The two practitioners (the distant site and the originating site) objected to having to split the single payment. Second, telehealth services covered under Medicare were limited to consultations. Third, originating sites were limited to practitioner's offices, hospitals, critical access hospitals, rural health clinics, and federally qualified health centers located in rural health professional shortage areas (HPSAs).

In 2001, the Benefits Improvement and Protection Act (BIPA) attempted to encourage telehealth by reducing regulations and increasing payments and covered telehealth services. First, BIPA removed the requirement that a practitioner be present at the originating site, so consulting providers no longer had to split the Medicare payment. Second, BIPA required Medicare to pay distant practitioners 100 percent of the rate under the physician fee schedule. That is, it required that Medicare pay distant practitioners the same amount for providing telehealth services that they would have received had they provided the same services in person. This payment to distant practitioners is adjusted by the geographic practice cost index of the site of the distant practitioner. Therefore, from the consulting physicians' perspective, their payment for telehealth care is equal to their payment for face-to-face care. Given equal Medicare payment for telehealth and faceto-face care, and the additional costs associated with telehealth, we would only expect telehealth adoption among providers with low opportunity costs of providing such services, and providers who are driven primarily by their service mission rather than by financial gain.

From the perspective of the payer (the Medicare program), BIPA required that Medicare make an additional facility fee payment to originating sites. This additional payment is currently $\$ 24$. Therefore, from the taxpayer's perspective, the telehealth visits costs $\$ 24$ more due to the additional facility fee paid to the originating site. In other words, the twentyfour dollars is the amount by which total payments for telehealth services exceed total payments for services provided in person. The net result is that the policy shifted from requiring two providers and making a single payment to requiring one provider and making two payments. The fee paid to originating sites continues to be adjusted annually for inflation and is not subject to any geographic payment adjustments. Finally, in 2001, BIPA expanded the range of telehealth services covered under Medicare to include office visits, individual psychotherapy, and pharmacological management.

Between 2003 and 2006, regulatory changes allowed the range of telehealth services covered under Medicare expanded further to include 
psychiatric diagnostic interview examinations, end-stage renal disease related services, and individual medical nutrition therapy. In 2008, the Medicare Improvements for Patients and Providers Act expanded the types of facilities eligible to be originating sites to include hospital-based and critical access hospital-based renal dialysis centers, skilled nursing facilities, and community mental health centers. In 2011, the range of covered telehealth services expanded again to include subsequent hospital and nursing care services, individual kidney disease education services, individual and group diabetes self-management training services, group medical nutrition therapy, and health and behavioral assessment and intervention.

Exhibit 1 summarizes the evolution of Medicare's payment policy to encourage adoption of telehealth by increasing payment, expanding coverage, and reducing provider requirements. In addition to these efforts, many grants to cover the costs of establishing telehealth systems were providedoverthelastdozenyearsbyfederalagencies, including the Centers for Medicare \& Medicaid Services (CMS), the Office for the Advancement of Telehealth, the National Telecommunications Infrastructure Administration, and the U.S. Department of Agriculture. These efforts reflect the long-standing hope that telehealth could be used to overcome a lack of local medical and surgical subspecialists in rural areas.

\section{Data and Methods}

Most telehealth research is based on case studies of a single telehealth site that is staffed by a few consulting specialists. The literature lacks a study that examines the full cross section of claims from all providers during a fiscal year. While CMS has presented data showing that the volume of Medicare FFS claims are slowly rising over time, they have not provided detailed information on providers or patients. In this study, we use a snap shot of a single years' claims data (2009) to take a closer examination of the telehealth providers and patients. By matching a full year's claims to individual patient ZIP codes and individual provider characteristics, we can get a closer descriptive study of telehealth in Medicare than in prior studies.

The primary data source for our analysis is $100 \%$ of telehealth Medicare claims for 2009, the most recently available data at the time of this study. These claims came from the Medicare carrier file. To identify telehealth claims from distant practitioners, we first pulled all claims with at least one "GT" modifier, which is used to indicate that the service was provided "via interactive audio and video telecommunications." Second, we excluded claims that had the GT modifier, but were not covered telehealth services under Medicare. These claims represented erroneous billing. Third, we excluded all claims for which the allowed charge was zero. The remaining claims-claims that had a GT modifier, were covered telehealth services (or at least billed as if they were covered services), and that yielded payment-represent our count of telehealth claims from distant practitioners. To identify telehealth claims from originating sites, we pulled claims with a HCPCS of "Q3014," which is used to bill for the "telehealth originating site facility fee," and among those claims, we included only those that had an allowed charge greater than zero. Lastly, we identified practitioners' specialty by merging the NPIs from the claims data with the NPIs from the Physician Master File for 2009, which includes information on practitioners' area of specialty. We also investigated the degree to which telehealth services are provided by physician assistants and nurse practitioners, which has not systematically been investigated in the past. By having individual provider information, we were 
Exhibit 1. Changes in Medicare Telehealth Policy Over Time

\begin{tabular}{|c|c|c|}
\hline Topic & $\begin{array}{c}\text { Initial policies from the Balanced } \\
\text { Budget Act of } 1997\end{array}$ & Policy changes \\
\hline Payment & $\begin{array}{l}\text { One payment: } \\
\text { Payment was set to the physician } \\
\text { fee schedule rate and split } 75-25 \\
\text { between the distant practitioner and } \\
\text { originating practitioner, respectively. } \\
\text { Total payment equaled the physician } \\
\text { fee schedule amount. }\end{array}$ & $\begin{array}{l}\text { Two payments: } \\
\text { On October 1, } 2001 \text { the Medicare, } \\
\text { Medicaid and SCHIP Benefits } \\
\text { Improvement and Protection Act of } 2000 \\
\text { (BIPA) required Medicare to pay distant } \\
\text { practitioners } 100 \text { percent of the rate under } \\
\text { the fee schedule and making a separate, } \\
\text { fixed payment to originating sites, even } \\
\text { if a practitioner was not present. The fee } \\
\text { paid to originating sites is currently } \$ 24 \text {. }\end{array}$ \\
\hline Originating site requirements & $\begin{array}{l}\text { Originating sites were required to } \\
\text { have a practitioner present with the } \\
\text { beneficiary. Originating sites had to } \\
\text { be located in rural health professional } \\
\text { shortage areas (HPSAs), and had to } \\
\text { be among the following facility types: } \\
\text { practitioner's office, hospital, critical } \\
\text { access hospital, rural health clinic, } \\
\text { and federally qualified health center. }\end{array}$ & $\begin{array}{l}\text { In 2001, BIPA removed the requirement } \\
\text { that originating sites have a practitioner } \\
\text { with the beneficiary. In addition, the } \\
\text { geographic areas in which originating } \\
\text { sites could be located were expanded to } \\
\text { include all areas outside of metropolitan } \\
\text { statistical areas. In 2008, the Medicare } \\
\text { Improvements for Patients and Providers } \\
\text { Act (MIPPA) expanded the types of } \\
\text { facilities eligible to be originating sites to } \\
\text { include hospital-based and critical access } \\
\text { hospital-based renal dialysis centers, } \\
\text { skilled nursing facilities, and community } \\
\text { mental health centers. }\end{array}$ \\
\hline Covered services & Only consultations were covered. & $\begin{array}{l}\text { In 2001, BIPA expanded coverage } \\
\text { to include office visits, individual } \\
\text { psychotherapy, and pharmacological } \\
\text { management. Between } 2003 \text { and } 2006 \text {, } \\
\text { a series of regulatory changes expanded } \\
\text { coverage to include psychiatric diagnostic } \\
\text { interview examinations, end-stage renal } \\
\text { disease related services, and individual } \\
\text { medical nutrition therapy by registered } \\
\text { dieticians. In 2008, MIPPA expanded } \\
\text { coverage to include subsequent hospital } \\
\text { and nursing care services, individual } \\
\text { kidney disease education services, } \\
\text { individual and group diabetes self- } \\
\text { management training services, group } \\
\text { medical nutrition therapy, and health and } \\
\text { behavioral assessment intervention. }\end{array}$ \\
\hline
\end{tabular}

SOURCE: Authors' analysis of legislative and regulatory changes. 
able to call individual providers who exhibited unusual billing patterns for telehealth patients living in urban areas.

\section{Key Findings}

Despite increases in Medicare payment rates for telehealth services and expansions of covered services, and reductions in provider requirements, the volume of telehealth services received by Medicare beneficiaries, although growing, remains low. Based on our examination of 2009 Medicare claims, we find that beneficiaries made about 38,000 telehealth visits in 2009. This is an increase from about 26,000 telehealth visits in 2006 (Hartstein et al., 2011). The $32 \%$ may sound large, but it can be misleading because it is starting from a very small base of 26,000 visits. The 12,000 visit increase $(26,000$ to 38,000$)$ represents one additional telehealth visit for every 700 rural Medicare beneficiaries. Only 369 practitioners submitted claims for 10 or more telehealth services to beneficiaries in 2009 . In addition, fewer than 14,000 beneficiaries had telehealth visits in 2009. These beneficiaries were dispersed across the countrywith almost 200 different counties being the resident of beneficiaries receiving 50 or more telehealth visits (Exhibit 2). Exhibit two highlights that only 14 counties had more than 300 visits among the county's residents, suggesting that the number of highly active telehealth sites is limited. Our claims data analysis is consistent with information obtained from rural-hospital site visits over the years where providers often have telehealth capability, but use it rarely.

Among these 38,000 visits, a material share appears to be billing for telehealth services that are not covered by Medicare under current policy. We found that for 81 of the 369 telehealth providers, a majority of their telehealth patients lived in urban areas. We contacted two practices that were billing for the most telehealth services to urban beneficiaries, representing roughly 4 percent of all 2009 claims. We found that both practices were errantly billing (and being paid) for video consultations with urban patients in their homes, which is not a covered service. The billing managers for these two practices told us they were not aware they could not bill for teleconferences in an individual's home and that they were not aware the individuals had to be located outside of an MSA to qualify for telehealth reimbursement. Therefore, our count of distant practitioners includes some practitioners who billed erroneously for telehealth services.

The number of claims from distant practitioners exceeded the number of claims from originating sites by a surprising amount. Among the 38,000 telehealth claims in 2009, about 16,000 claims do not have a bill from an originating site (e.g., rural hospital), as is allowed by Medicare. A share of the 16,000 claims are errant billings by the consulting physician as was the case for the two physician practices we contacted. A share of the 16,000 could also be cases in which the distant site chose not to bill for the $\$ 24$.

Of the relatively small number of telehealth services received by Medicare beneficiaries, the most common are mental health services, including pharmacological management. We found that of the 38,000 telehealth visits that Medicare beneficiaries had in 2009, most visits (62 percent) were for mental health services: pharmacological management (47 percent), individual psychotherapy (8 percent), and psychiatrist diagnostic interview examinations (7 percent). About one third (31 percent) were office and other outpatient visits (Exhibit 3). Five percent were for end-stage renal disease consultations. The remaining 2 percent were for other services.

Similarly, as shown in Exhibit 4, we found that of the 369 distant practitioners who provided 10 or more Medicare-covered telehealth services in 2009 , roughly half (49 percent) were mental health practitioners: psychiatrists (44 percent), clinical 
Exhibit 2. Geographic Distribution of Medicare Beneficiaries Using Telehealth

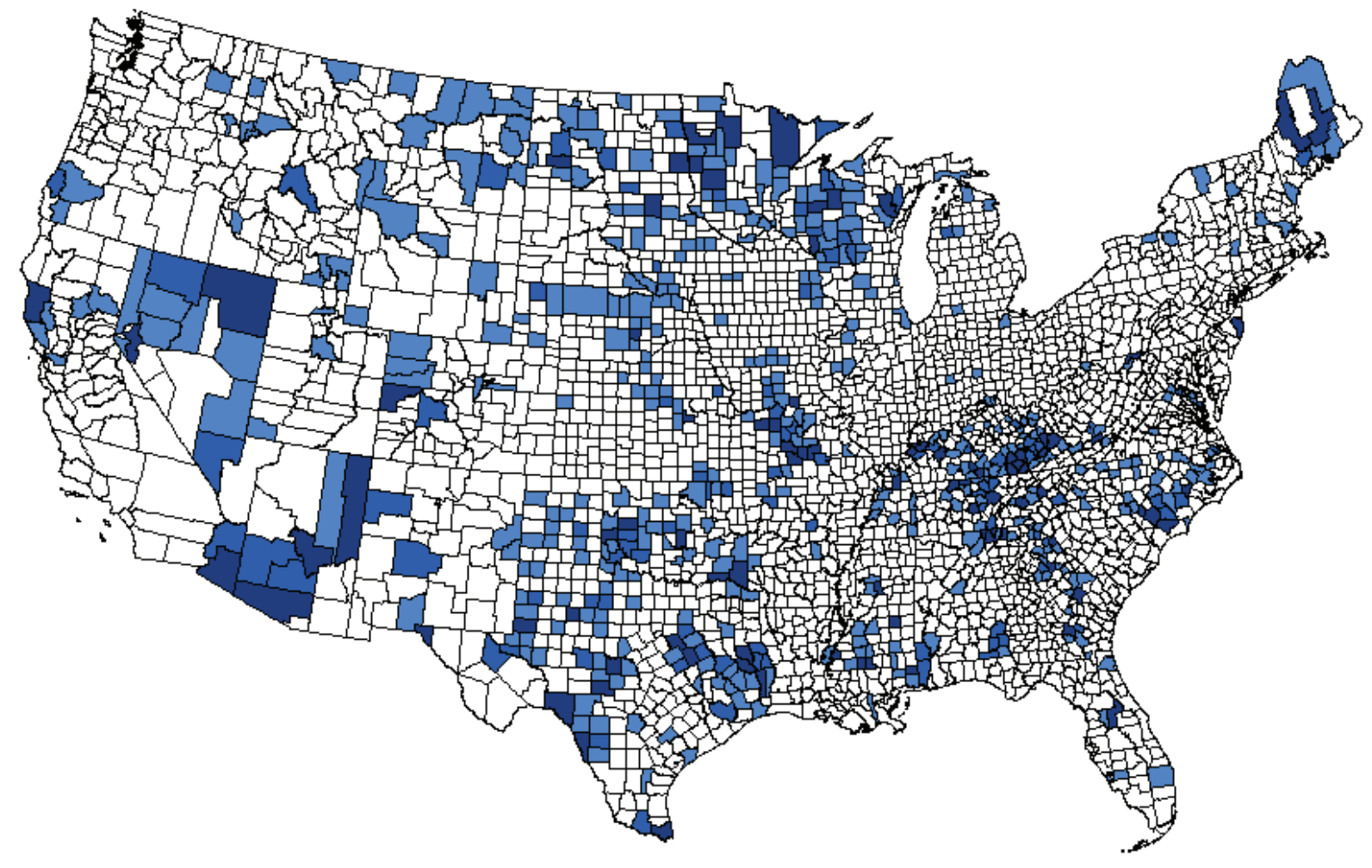

Telehealth visits by beneficiary's county of residence

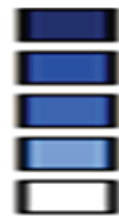

$\begin{array}{ll}301-983 & (14) \\ 101-300 & (77) \\ 51-100 & (97) \\ 11-50 & (409) \\ 0-10 & (2623)\end{array}$

SOURCE: Authors' analysis of patient ZIP code data from Medicare claims and Medicare enrollment files.

psychologists (3 percent) and licensed clinical social workers ( 2 percent). About one third (32 percent) were physicians of specialties other than mental health, such as family practice and internal medicine subspecialists, nephrologists, and neurologists. Additionally, we found that about one-in-five (19 percent) were non-physician professionals: nurse practitioners (13 percent), physician assistants (3 percent) and certified clinical nurse specialists ( 3 percent). ${ }^{1}$

\footnotetext{
${ }^{1}$ For the purposes of our paper, we chose not to categorize licensed clinical social workers as non-physician professionals, but instead as mental health practitioners.
}

Given that telehealth is regarded as a means to compensate for shortages of certain subspecialist physicians in rural areas, we found it surprising (1) that we did not see greater telehealth adoption among physicians across medical and surgical subspecialties and (2) that we saw a significant share of telehealth services were being provided by non-physician professionals. Yet these findings are consistent with the economic-model explanations for the low uptake of telehealth mentioned earlier: the theory that specialist physicians already have sufficient in-person patient populations and therefore consider telehealth, which involves 
Exhibit 3. Type of Telehealth Service Provided

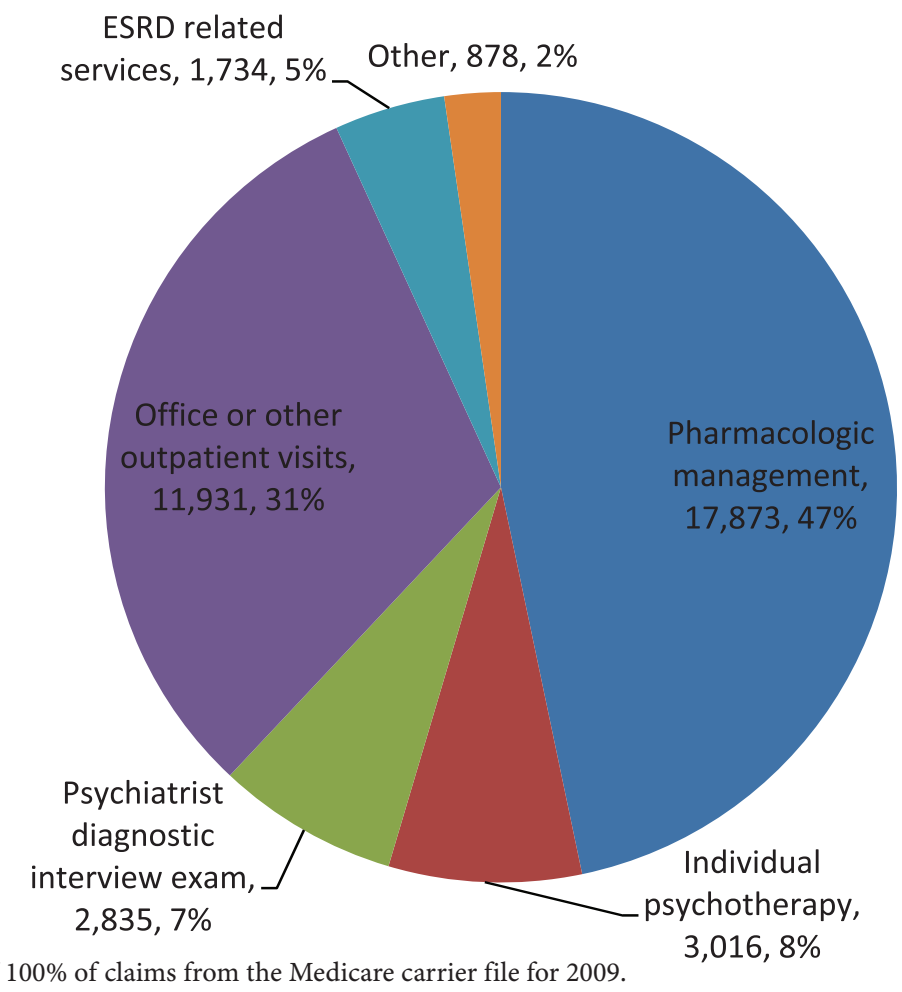

SOURCE: Authors' analysis of $100 \%$ of claims from the Medicare carrier file for 2009.

added time for them, to be financially unattractive. In other words, specialist physicians' opportunity cost of providing telehealth services may be too high, which could explain why so few specialist physicians provide Medicare-covered telehealth services. Therefore, specialist physicians who choose to participate in telehealth may be doing so as part of their service mission and a desire to integrate care across regions, rather than due to the financial benefits. Non-physician professionals, on the other hand, are likely to have an opportunity cost of providing telehealth that is lower than that of specialist physicians. Nonphysician professionals' lower opportunity cost of providing telehealth services could explain why we find that a surprisingly significant share

Exhibit 4. Practitioners Providing 10 or More Telehealth Services in 2009

\begin{tabular}{lcc}
\hline Type of practitioner & Count & Percent \\
\hline Psychiatrists, psychologists, clinical social workers & 181 & 49 \\
NPs, PAs, clinical nurse specialists & 69 & 19 \\
Family practice, internal medicine specialists & 24 & 7 \\
Nephrologists & 14 & 4 \\
All other (e.g., neurologists, cardiologists, hematologists, etc.) & 81 & 22 \\
Total & 369 & \\
\hline
\end{tabular}

NOTES: NP (nurse practitioner), PA (physician assistant). Figures do not sum to 100 percent due to rounding. It is possible that our counts of NPs, PAs, clinical nurse specialists, and clinical social workers are undercounts, because these practitioners sometimes bill for services under the names of the physicians they work for.

SOURCE: Authors' analysis of $100 \%$ of claims from the Medicare carrier file for 2009. 
(about one-in-five) of medical professionals who provide Medicare-covered telehealth services are non-physicians.

\section{Limitations}

It is possible that our count of telehealth services, our count of beneficiaries receiving such services, and our count of practitioners providing such services are undercounts, because the "GT" modifier, which we used to identify telehealth services, is not tied to payment. This means practitioners could have billed for a telehealth service, but not have included the GT modifier in their claim. In addition, our analysis did not include claims for test interpretations that do not require the GT modifier. It is also possible that our counts of nurse practitioners, physician assistants, clinical nurse specialists, and clinical social workers are also undercounts, because these practitioners sometimes bill for services under the names of the physicians they work for. The extent of these potential undercounts is unknown.

The data for this paper consists of fee-forservice claims for the types of telehealth currently paid for by Medicare. Therefore, we are not able to evaluate the value of telehealth in other situations, such as home monitoring, or in new delivery systems, such as accountable care organizations, or in capitated MA plans. ACOs are too new to have claims on our 2009 data set, and MA encounter claims were not available at the time this paper was written. It is possible that some ACOs or MA plans may start to use telehealth as a substitute for faceto-face care. Future research is needed to evaluate the extent to which ACOs are able to benefit from these models.

There are few independent evaluations of the outcomes associated with the new areas of telehealth, such as tele-emergency care. We were not able to evaluate these types of telehealth in this study due to there not being any specifying data on Medicare claims to indicate if a telehealth consultation was done in an emergency situation. There is a need for further research on teleemergency care by independent researchers who do not work in the programs being evaluated.

\section{Discussion}

Despite the changes in Medicare payment policy over the past 15 years that sought to encourage telehealth by increasing payment rates, expanding coverage, and reducing provider requirements, Medicare beneficiaries rarely receive covered telehealth services. One possible explanation for this finding is a willingness of patients to drive for most of the services covered as telehealth by Medicare. Another could be a general lack of interest among practitioners, possibly owing to sufficient face-to-face patient populations and the desire to avoid the hassles associated with changing practice patterns. Of the relatively few telehealth services provided to Medicare beneficiaries, the most common services are mental health services, including pharmacological management. With the literature suggesting that mental health care provided via telehealth can be equally as effective as mental health care provided in person, providing these types of services seems to be appropriate. We also see a significant number of lower-cost physician assistants and nurse practitioners providing Medicare-covered telehealth services.

While telehealth care can improve access for isolated rural beneficiaries, it has also been used to provide in-home care for urban individuals who could not travel for face-to-face care. For some of these patients who are in close proximity to a provider who can provide face-to-face visits, the additional costs associated with telehealth visits may not be justified. 
For there to be a viable business model for telehealth, the benefit of telehealth for the patient must exceed the cost of telehealth for the provider. From the standpoints of the patient and society, paying an amount that is above the benefit to the patient does not make sense. The clearest benefit of telehealth for rural patients (apart from the benefit of improved health) is the benefit of reduced travel, which saves the patient both time and money. Especially for patients in remote rural areas, the value of reduced travel can be considerable. Sometimes there are additional benefits of telehealth for the patient, as is the case with tele-emergency care, which makes emergency medicine expertise and support available to patients in emergency situations where travel is not possible. However, in cases where face-to-face care is an option, it may be that the only tangible benefit of telehealth is reduced travel. In these cases, telehealth only makes sense if the patients' travel-cost savings exceed the provider's additional time and expense of providing the visit. This can be a difficult hurdle when the billing rate of the provider (e.g., dermatologist) is high relative to the value that rural patients place on avoiding travel costs. The benefit of telehealth for the patient is more likely to exceed the cost of telehealth for the provider when the provider's opportunity cost of providing telehealth services is comparatively low. This may explain why we see that disproportionate shares of Medicare-covered telehealth services are being provided (1) by mental health care providers, who (because of the nature of their services) can provide telehealth care with low additional time and expense relative to face-to-face care, and (2) by non-physician professionals, whose earning potentials per a unit of time are lower than that of specialist physicians.

Ultimately, growth in telehealth may depend on two factors: the benefit to the patient (e.g., reduced travel and improved outcomes), the net cost (including opportunity cost) to the provider. If a healthcare system has excess capacity, they may be willing to subsidize telehealth to bring additional patients into the regional system of care and potentially increase their volume of profitable face-to-face care. From the specialist perspective, the net cost of the telehealth service will be the cost of providing telehealth services less the marginal profits on the increased volume of face-to-face visits. The marginal profit of gaining market share in rural areas will only be positive if the specialty practice has a shortage of face-to-face patients.

There may be potential growth for telehealth in situations in which the benefit to the patient exceeds the net cost to the provider. These include emergency situations in which travel is not possible or exceedingly difficult. For ER patients and inpatients in need of emergency care in isolated rural settings, the benefit of telehealth may be great enough to justify the cost to the provider. ${ }^{2}$ There also may be potential growth for telehealth in the relatively few situations in which the cost (including opportunity cost) of providing telehealth services is comparatively low, such as mental-health visits and visits with non-physician professionals. Another example is telepharmacy prescription fills for patients in communities without 24 hour pharmacy coverage who need telehealth review of medications. Because pharmacy technicians staffing remote pharmacy sites have lower opportunity costs compared to their supervising pharmacists at central sites, telepharmacy has been shown to be a more viable alternative than a freestanding pharmacy. In these cases the cost of telepharmacy is fully supported by the patients' payments for pharmaceuticals. To summarize, the extent to which the benefit to the patient is great (in terms of reduced travel for essential health care) and the cost to the provider is

\footnotetext{
${ }^{2}$ These costs would be allowable expenses for critical access hospitals, which receive cost-based reimbursement under Medicare.
} 
comparatively low, reflects the extent to which there are opportunities for growth in telehealth. In some cases it will be socially desirable to expand telehealth services, but only in cases where the value of emergency access or reduced patient travel time outweighs the additional cost of delivering care via telehealth.

\section{Acknowledgment}

The authors would like to acknowledge Mark Miller and Carlos Zarabozo for their insights as we worked through this study.

\section{Correspondence}

Jeff Stensland, Ph.D., Medicare Payment Advisory Commission, 601 New Jersey Avenue, N.W. , Washington, DC 20001, jstensland@medpac.gov, Tel. 202-220-3726.

\section{References}

Abel, R. L., Warren, K., Bean, G., Gabbard, B., Lyder, C. H., Bing, M., \& McCauley, C. (2005). Quality improvement in nursing homes in Texas: Results from a pressure ulcer prevention project. Journal of the American Medical Directors Association, 6(3), 181-188. PubMed http://dx.doi.org/10.1016/j.jamda.2005.03.011

Blanchet, K. D. (2008). Innovative programs in telemedicine. The Telemedicine Program at Fletcher Allen Health Care and the University of Vermont College of Medicine. Telemedicine and e-Health, 14(2), 122-126.

Doheny-Farina, S., Callas, P. W., Ricci, M. A., Caputo, M. P., Amour, J. L., \& Rogers, F. B. (2003). Technical communication and clinical health care: Improving rural emergency trauma care through synchronous videoconferencing. Potsdam, NY: Clarkson University. http:// ntiaotiant2.ntia.doc.gov/top/docs/eval/ pdf/506099024e.pdf
Duchesne, J. C., Kyle, A., Simmons, J., Islam, S., Schmieg, R. E., Jr., Olivier, J., \& McSwain, N. E., Jr. (2008). Impact of telemedicine upon rural trauma care. The Journal of Trauma, 64(1), 92-97.PubMed http://dx.doi.org/10.1097/TA.0b013e31815dd4c4

Grigsby, B., Brega, A. G., Bennett, R. E., Devore, P. A., Paulich, M. J., Talkington, S. G. ... Grigsby, J. (2007). The slow pace of interactive video telemedicine adoption: the perspective of telemedicine program administrators on physician participation. Telemedicine and $e$-Health 13(6), 645-656.

Hartstein, M., Warren, J., \& Howe, R. (2011). Medicare telehealth [Powerpoint presentation].

Hersh, W. R., Hickam, D. H., Severance, S. M., Dana, T. L., Krages, K. P., \& Helfand, M. (2006). Telemedicine for the Medicare population: Update (AHRQ Report No. 131). Rockville, MD: Agency for Healthcare Research and Quality.

Institute of Medicine 2004. Quality through collaboration: The future of rural health care. Washington, DC: National Academies Press.

Johnston, B., Weeler, L., Deuser, J., \& Sousa, K. H. (2000). Outcomes of the Kaiser Permanente Tele-Home Health Research Project. Archives of Family Medicine, 9(1), 40-45. PubMed http:// dx.doi.org/10.1001/archfami.9.1.40

Latifi, R., Weinstein, R. S., Porter, J. M., Ziemba, M., Judkins, D., Ridings, D., . . Leyva, F. (2007). Telemedicine and telepresence for trauma and emergency care management. Scandinavian Journal of Surgery, 96(4), 281-289. PubMed

Luo, J. S. (2008). Telemedicine: Is it time now? Primary Psychiatry, 16(2), 27-30.

McCarthy, D., Nuzum, R., Mika, S., Wrenn, J., \& Wakefield, M. (2008). The North Dakota experience: Achieving high-performance health 
care through rural innovation and cooperation. New York, NY: The Commonwealth Fund.

O’Reilly, R., Bishop, J., Maddox, K., Hutchinson, L., Fisman, M., \& Takhar, J. (2007). Is telepsychiatry equivalent to face-to-face psychiatry? Results from a randomized controlled equivalence trial. Psychiatric Services (Washington, D.C.), 58(6), 836-843. PubMed http://dx.doi.org/10.1176/ appi.ps.58.6.836

Ricci, M. A., Caputo, M., Amour, J., Rogers, F. B., Sartorelli, K., Callas, P. W., \& Malone, P. T. (2003). Telemedicine reduces discrepancies in rural trauma care. Telemedicine Journal and e-Health, 9(1), 3-11. PubMed http://dx.doi. org/10.1089/153056203763317602

Richardson, L. K., Frueh, B. C., Grubaugh, A. L., Egede, L., \& Elhai, J. D. (2009). Current directions in videoconferencing tele-mental health research. Clinical Psychology: Science and
Practice, 16(3), 323-338. PubMed http://dx.doi. org/10.1111/j.1468-2850.2009.01170.x

Rogers, F. B., Ricci, M., Caputo, M., Shackford, S., Sartorelli, K., Callas, P.,... Daye, S. (2001). The use of telemedicine for real-time video consultation between trauma center and community hospital in a rural setting improves early trauma care: Preliminary results. The Journal of Trauma, 51(6), 1037-1041. PubMed http://dx.doi. org/10.1097/00005373-200112000-00002

Sorondo, R. J. G. Z. B., Holmberg, R., \& Bjorn, P. (2011). Telemedicine consultation for emergency trauma: The 130 million square foot trauma room. Bulletin of the American College of Surgeons, 96(6), 12-19.

Whitten, P., \& Buis, L. (2006). Private payer reimbursement for telemedicine services in the United States. East Lansing, MI: Michigan State University. 